\title{
The Orbital Origins of Chemical Bonding in Phase-Change Materials
}

\author{
Jan Hempelmann, Peter C. Müller, Christina Ertural, and Richard Dronskowski*
}

J. Hempelmann, P.C. Müller, C. Ertural

Institute of Inorganic Chemistry, RWTH Aachen University, D-52056 Aachen, Germany.

Prof. Dr. R. Dronskowski

Institute of Inorganic Chemistry, RWTH Aachen University, D-52056 Aachen, Germany. Jülich-Aachen Research Alliance (JARA-CSD), RWTH Aachen University,

D-52056 Aachen, Germany

Hoffmann Institute of Advanced Materials, Shenzhen Polytechnic, 7098 Liuxian Blvd, Nanshan District, Shenzhen 518055, China

E-mail: drons@HAL9000.ac.rwth-aachen.de

Keywords: phase-change materials, metavalent bonding, multicenter bonding, wavefunction analysis

ABSTRACT: Layered phase-change materials in the $\mathrm{Ge}-\mathrm{Sb}-\mathrm{Te}$-system are widely used in data storage and are the subject of intense research to understand the elusive quantum-chemical origin of their unique properties. To uncover the nature of the underlying periodic wavefunction, we study the interacting atomic orbitals including their phase information as revealed by crystal orbital bond index (COBI) and fragment crystal orbital (FCO) analysis. In full accord with previous and also new findings based on projected force constants $(\mathrm{pFC})$, we demonstrate the decisive role of multicenter bonding along straight atomic connectivities such as $\mathrm{Te}-\mathrm{Ge}-\mathrm{Te}$ and $\mathrm{Te}-\mathrm{Sb}-\mathrm{Te}$. While the here found multicenter bonding resembles well-established three-center four-electron bonding in molecules, its solid-state manifestation beyond a molecular motif leads to distinct longe-range consequences, thus serving to contextualize the aforementioned material properties usually termed "metavalent". For example, we suggest multicenter bonding to be the origin of their astonishing bond-breaking and also phase-change behavior. As a hole-in-one, 
multicenter bonding immediately explains the too small "van der Waals" gaps between individual layers since multicenter bonding forces these gaps to shrink below the nonbonding Te-Te distances.

\section{Introduction}

Phase-change materials (PCM) have been an intensely discussed research subject for at least six decades. ${ }^{[1]}$ Due to their unusual ability to quickly and reversibly switch between an amorphous and a physically distinguishable crystalline state, PCM find application in both optical and resistive memory storage devices in which the two phases are used to write and read the data. ${ }^{[2]}$ Representatives of this material class are commonly found to comprise those compounds that violate the $8-N$ rule by a certain electron surplus, with main-group IV monotellurides such as $\mathrm{GeTe}, \mathrm{SnTe}$ or $\mathrm{PbTe}$ being prototypical; note that the corresponding electron-precise $8-N$ representatives $\mathrm{CaTe}, \mathrm{SrTe}$, and BaTe (with noble-gas configurations for all atoms) behave unremarkably. Despite almost insignificant electronegativity differences, ${ }^{[3]}$ the rocksalt type and related structures - usually found in ionic compounds - are common for GeTe, SnTe, and PbTe for which tetrahedral coordination (with shorter bond distances needed for covalent bonding) would look more typical, at least at first sight. ${ }^{[4]}$

Given the set of unusual physical characteristics alluded to before and the significance of PCM for material science, it is hardly surprising that categorizing existing PCM and the search for even better ones has been the subject of vigorous research. As for now, the sum of all empirical knowledge has coalesced into a unique set of properties, often referred to as their property portfolio ${ }^{[5]}$ which typically includes ${ }^{[6]}$ an extraordinarily large optical dielectric constant, an unusually large electrical conductivity, and a large Grüneisen parameter. From a more chemical point of view, the high probability for multiple emission events in atom-probe tomography $(\mathrm{APT})^{[7]}$ is worth mentioning, so far interpreted as atoms "sticking together" even after having been ripped out from the bulk. The most obvious and indisputable structural property is given 
by the too small van-der-Waals gaps in layered PCM systems which refuses a convincing explanation until today. ${ }^{[8]}$

The aforementioned property set has proven to be an apt identifier for phase-change behavior although its origins have so far eluded an in-depth bonding analysis based on orbitals. Nonetheless, it has been possible to empirically $\operatorname{map}^{[9]}$ various materials including PCM based on an electron-density partitioning scheme and thus derived descriptors for ionicity (electrons transferred, ET) and covalency (electrons shared, ES), thereby identifying prominent PCM as being positioned between the covalent and metallic bonding regimes. Ionicity seems to be negligible with near-zero electronegativity differences ${ }^{[3]}$ which is reflected in small values of Mulliken and Löwdin charge for the respective atoms. ${ }^{[10,11]}$ Consequently, the metavalent bonding term looks fitting for a region between (and also different from) metals and prototypical covalent materials.

In what follows, we will provide quantum-chemical evidence, by an orbital-based analysis of the underlying PCM wavefunction, that the mechanistic cause of metavalent bonding is the solid-state analog of multicenter bonding known from molecules violating the $8-N$ rule. As such, this study complements a previous theoretical PCM-related discovery, in particular the occurrence of unique long-range projected force constants (pFC, also known as bond projected force constants $\mathrm{BPFC}{ }^{[12]}$ ) detected in rocksalt type chalcogenide $\mathrm{PCM} \cdot{ }^{[10]}$ In the spirit of a molecular quantum-chemical approach, we apply crystal orbital bond index (COBI) analysis to detect pairwise but also multicenter interactions, previously inaccessible for solids, ${ }^{[13]}$ to be compared with fragment crystal orbital (FCO) decomposition. ${ }^{[14]}$ Eventually, both COBI/FCO as well as pFC strongly suggest the same bonding situation when applied to the relevant GeTe$\mathrm{Sb}_{2} \mathrm{Te}_{3}$ (often dubbed "GST") pseudobinary system.

In its various compositions, the latter system is among the most prominent phase-change materials in use today. ${ }^{[15]}$ GST is intimately related to $\mathrm{Sb}_{2} \mathrm{Te}_{3}$ (Figure 1) which crystallizes in the layered rocksalt-derived $\mathrm{Bi}_{2} \mathrm{Te}_{3}$-type, ${ }^{[16]}$ with bond angles close to $90^{\circ}$ and almost equal $\mathrm{Sb}-$ 
Te bond lengths but separated into five-atom thick "quintuple" layers ( $\mathrm{Te}-\mathrm{Sb}-\mathrm{Te}-\mathrm{Sb}-\mathrm{Te})$. $\mathrm{Sb}_{2} \mathrm{Te}_{3}$ can also be chemically "expanded" into the entire GST material family (Figure 1) with compounds such as $\mathrm{Ge}_{1} \mathrm{Sb}_{2} \mathrm{Te}_{4}$ (GST124), $\mathrm{Ge}_{2} \mathrm{Sb}_{2} \mathrm{Te}_{5}$ (GST225) and $\mathrm{Ge}_{3} \mathrm{Sb}_{2} \mathrm{Te}_{6}$ (GST326). In their most stable structural variant, Ge is inserted in the center of the quintuple layer, effectively increasing the layer thickness by two atoms for each GeTe formula unit added. ${ }^{[17]}$ Naturally, the more GeTe is added, the more the resulting phase approaches pure GeTe which crystallizes in the stable Peierls distorted $\alpha-G e T e$ as well as the metastable rocksalt type $\beta$-GeTe structure. ${ }^{[18]}$ The somewhat mysterious structural gap between two layers (that is, between the terminal Te atoms) has often been referred to as a van-der-Waals (vdW) gap, for obvious reasons, but a closer look reveals that this gap is significantly (12\%) smaller than twice the sum of the vdW radius. ${ }^{[8]}$ Because there is no external pressure in the GPa range, there must be more than simple vdW forces; indeed, previous projected COHP analysis has already detected small but significant covalency across the gap. ${ }^{[1]}$ The latter stems from multicenter bonding, as elaborated below, present in the entire set of phase-change materials studied here.

\section{Theory}

As ingeniously recognized in 1927 already ${ }^{[19]}$ by means of valence-bond theory, covalent bonding is a quantum-chemical interference phenomenon in which the interacting atomic wavefunctions (but not the electron density) interact either constructively (bonding) or destructively (antibonding), with all consequences for the resulting molecular wavefunction and its energetics. In modern molecular-orbital language, ${ }^{[20]}$ the hydrogen molecule $\mathrm{H}_{2}$ then results from two $1 \mathrm{~s}$ atomic orbitals constructively overlapping to give the bonding $\sigma_{\mathrm{g}}$ molecular orbital in which the two electrons are "stored" and yield an $\mathrm{H}-\mathrm{H}$ single bond as well as the He noblegas configuration for both $\mathrm{H}$ atoms; the antibonding $\sigma_{\mathrm{u}}{ }^{*}$ molecular orbital stays unoccupied, as sketched in Figure 2 a/b by a DOS and COBI (see below) plot. Essentially the same orbitalwise mechanism (only slightly more complicated due to more atomic orbitals) is found for diatomics 
such as $\mathrm{N}_{2}$, larger molecules such as $\mathrm{C}_{2} \mathrm{H}_{6}$, and even for proteins of any size, all of them fulfilling the octet $(8-N)$ rule. In case the latter rule is not fulfilled, multicenter bonding sets in, as has been established in molecular quantum chemistry for decades already, be it electrondeficient 3-center-2-electron (3c2e) bonds in $\mathrm{B}_{2} \mathrm{H}_{6}{ }^{[21]}$ or electron-rich 3-center-4-electron (3c4e) bonds in $\mathrm{XeF}_{2} ;{ }^{[22,23]}$ other scenarios have also been reported. ${ }^{[24]}$ For the case of $\mathrm{XeF}_{2}$, its electronic structure has also been sketched in Figure $2 \mathrm{c} / \mathrm{d} / \mathrm{e}$. The existence of $\mathrm{XeF}_{2}$ is due to $\sigma$ type molecular orbitals formed by the p-orbitals along the bonding direction ${ }^{[23]}$ but which also include an s-orbital contribution. ${ }^{[25]}$ Despite $\mathrm{Xe}$ in $\mathrm{XeF}_{2}$ having a valence electron count of 10 instead of 8 , the final molecular orbitals are energetically lowered compared to the atomic orbitals, and there results a bonding interaction and a stable molecule. A detailed and even quantitative analysis of this bonding mechanism can be carried out by means of a generalized bond index for molecules ${ }^{[26]}$ resembling the original Wiberg-Mayer idea. ${ }^{[27]}$ The new crystal orbital bond index $(\mathrm{COBI})^{[13]}$ depicted in Figure 2 for molecular $\mathrm{H}_{2}$ and $\mathrm{XeF}_{2}$ not only serves as the periodic (solid-state) Wiberg-Mayer equivalent, it also allows to analyze three-center (four-center, five-center, etc.) in addition to pairwise interactions in both molecules and solids. For example, the three-center $\mathrm{COBI}^{(3)}$ is formed as an averaged product of density matrices involving atomic orbitals $(\mu, v, \chi)$ on three atoms and all their combinations: ${ }^{[13]}$

$$
\mathrm{COBI}^{(3)}=P_{\mu \nu} P_{\nu \chi} \sum_{j, \mathbf{k}} w_{\mathbf{k}} \operatorname{Re}\left(c_{\chi, j \mathbf{k}}^{*} c_{\mu, j \mathrm{k}}\right) \cdot \delta\left(\varepsilon_{j}(\mathbf{k})-E\right)
$$

The solid-state calculus is performed for all bands $j$ at all $\mathbf{k}$ values. While the 2 -center COBI is quite intuitive as it translates to the chemical bond order, interpreting a multicenter bonding indicator is non-trivial. From molecular applications, it is well-known that negative numbers for the three-center bond index are found for electron-rich interactions while positive values are found for systems with an electron deficit. Hence, Figure 2 d indicates a 0.49 two-center bond order for $\mathrm{Xe}-\mathrm{F}$ based on the integrated $\mathrm{COBI}^{(2)}$ (ICOBI) value, in addition to a significant -0.32 three-center $\mathrm{F}-\mathrm{Xe}-\mathrm{F}$ integrated $\mathrm{COBI}^{(3)}$, thereby highlighting the multicenter bonding in 
$\mathrm{XeF}_{2}$. In addition to two- and three-center interactions, there are also nonbonding contributions (black spikes in the DOS plot in Figure 2c); we reiterate that such distinction between bonding, antibonding, and nonbonding is only possible through the orbital-phase information. All bonding contributions are given in red and illustrated by orbitals sketches, to be compared with Figure $2 \mathrm{~d} / \mathrm{e}$. COBI in all its variants is part of LOBSTER ${ }^{[28]}$ which allows for chemical-bonding analysis within a local-orbital framework but based on plane wave simulations.

\section{Results and Discussion}

\subsection{The Crystal Orbital Bond Indices and the Projected Force Constants of GeTe}

For reasons that will become obvious in what follows, we first revisit rocksalt-type GeTe for which evidence for multicenter interactions has already been demonstrated, but via projected force constants, ${ }^{[10]}$ that is, from the phononic point of view. Electronically, there have also been indications for multicenter bonding, indirectly so. An earlier COHP bonding analysis in Gedefect rocksalt-type GeTe already showed that an intermediate Ge atom between two Te atoms leads to an increase of the Te-Te bond energy (Kohn-Sham part) by a factor of almost 40; without the in-between Ge atom, there is almost no Te-Te interaction, so some kind of multicenter interactions must be present. ${ }^{[29]}$ On purpose, we will now focus on such three-center interactions. Fortunately, due to the rocksalt type's high symmetry and p-orbital orthogonality, there are only two different kinds of three-center interactions to consider: one that spans from

Te to Te, mediated by Ge, conveniently written as $\mathrm{Te}$ Ge $\mathrm{Te}$ in our notation. Inversely, there is another one that spans from $\mathrm{Ge}$ to $\mathrm{Ge}$ and is mediated by Te; we formulate this as $\mathrm{Ge}-\mathrm{Te}-\mathrm{Ge}$. For visual analysis, orbitalwise three-center COBI plots, their integrated values und how they combine for the total three-center interaction are depicted in Figure 3. Because there is no clear separation of molecule-like units in $\beta$-GeTe (unlike $\alpha$-GeTe), assuming that such three-center bonds exist as discrete entities seems unfitting. Likewise, the interaction range is not necessarily 
limited to three centers, so we are likely looking at partial contributions to the total multicenter interaction across $n$ centers as suggested previously. ${ }^{[10]}$ Quite obvious to the naked eye, the COBI plots in Figure 3 are qualitatively similar to each another, showing substantial orbital interaction across the same energy range. $p_{x} \frac{p_{x}}{p_{x}}$ (Figure $3 a / e$ ) was already suggested as the main component to multicenter bonding, as confirmed by $\mathrm{COBI}^{(3)}$ but $\mathrm{s} \stackrel{\mathrm{p}_{\mathrm{x}}}{\mathrm{p}_{\mathrm{x}}}$ also makes significant contributions. Notably, for Te - Ge $\mathrm{Te}$ the two orbital contributions add up to a net ICOBI of -0.10 , whereas the ones for $\mathrm{Ge} \stackrel{\mathrm{Te}}{-} \mathrm{Ge}$ compensate each other to yield a zero ICOBI value. That is to say that the in-between Ge atom (with a filled $4 \mathrm{~s}^{2}$ configuration, locally deviating from the octet rule) is mediating the multicenter bond but in-between Te (with a formal Xe configuration, octet rule fulfilled) does not amount to a net bonding interaction. As such, the result is not too surprising but the simplified fragment crystal orbital (FCO) diagrams on the right (Figure $3 \mathrm{~d} / \mathrm{h}$ ) further elucidate the bonding situation. In the $\mathrm{FCO}$, the entire set of orbital interactions is represented as resulting from two interacting fragments (Ge and TeTe in Figure 3d), similar to a fragment molecular orbital diagram. ${ }^{[30]}$ To keep interpretation simple for the extended solid, however, we model the TeTe fragment by the two individual Te atoms, rather than a single entity (which would need another diagonalization of the respective Hamiltonian). Figure $3 \mathrm{~d} / \mathrm{h}$ evidences that there is significant orbital overlap, the individual $4 s / 4 p$ and $5 s / 5 p$ levels being very close in energy, thereby rationalizing the insignificant amount of charge transfer mentioned before; these are truly non-ionic materials.

For the Ge-mediated interaction in Figure $3 \mathrm{a}-\mathrm{d}$, the negative ICOBI may be understood just like multicenter bonding for molecules. GeTe with a valence-electron concentration of 5 (not 4 as for the $8-N$ equivalent $\mathrm{CaTe}$ ) is an electron-rich system mediated by p-orbitals and therefore exhibits an electron-rich multicenter bond, manifesting in a negative ICOBI. Its absolute value $(-0.099)$ along one direction only is notably smaller than for the molecular case but this is to be expected: multicenter bonding occurs along three perpendicular directions and there may also 
be multicenter interactions with an even wider range beyond three centers. For molecular $\mathrm{XeF}_{2}$, everything stops with the one-dimensional three-center case and $\operatorname{ICOBI}^{(3)}=-0.32$.

For the $\mathrm{Ge} \stackrel{\mathrm{Te}}{-} \mathrm{Ge}$ case, the peculiarities of periodic bulk materials become significant. Despite comparable degrees of orbital interaction below the Fermi level (evident by the non-zero values of the plots in Figure $3 \mathrm{e} / \mathrm{f}$ ), the $\mathrm{COBI}^{(3)}$ integral amounts to a zero value (Figure $3 \mathrm{~g}$ ), as said before. By means of the fragment crystal orbital diagram, this very electronic state resembles a three-center equivalent of a nonbonding interaction. In molecular-orbital theory, nonbonding states may indicate the presence of lone-pairs, as they do in the classical case of the dinitrogen molecule. While it is unlikely to be entirely analogous within the context of a three-center interaction - the degree of localization across the centers is unclear after all - the nature and existence of lone-pairs within the metavalently bonded family of materials has been vividly discussed in the past. ${ }^{[31,32]}$ That is to say that the notion of a (directionally) partially delocalized “quasi lone-pair" as we may understand a three-center equivalent, is not unfounded.

Remarkably, while the two ICOBI values for $\mathrm{Te} \stackrel{\mathrm{Ge}}{-} \mathrm{Te}$ and $\mathrm{Ge} \stackrel{\mathrm{Te}}{\mathrm{Ge}}$ are vastly different, the corresponding projected force constants $(\mathrm{pFC})$ are almost the same, with the nonbonding threecenter interaction even being larger in size. We reiterate that $\mathrm{pFC}$ have been demonstrated to indicate multicenter interactions in GeTe. ${ }^{[10]}$ While they rest on an entirely different theoretical background as regards forces and phonons, the resulting pFC easily quantify the interaction strength between any two atoms in a supercell. For the case of GeTe, extraordinarily strong force constants for atoms being very far apart suggested multicenter bonding as a possible explanation.

Coming back to the vanishing $\mathrm{Ge}-\mathrm{Te}$ Ge three-center interaction, the electronic state is seemingly nonbonding in terms of orbital interactions (because they all cancel), resulting in no lowering of the energy for this state. And yet, the linear configuration is still so sensitive to geometric perturbation (resulting in a high $\mathrm{pFC}$ ) that there is a collective net contribution of that 
nonbonding scenario which also supports the metastability of $\beta$-GeTe and the orthogonal linear chains facilitating multicenter $\mathrm{Te}-\mathrm{Ge}-\mathrm{Te}$ interactions.

As an in-between summary, the $\mathrm{Te} \stackrel{\mathrm{Ge}}{-} \mathrm{Te}$ and $\mathrm{Ge} \stackrel{\mathrm{Te}}{-} \mathrm{Ge}$ examples illustrate how COBI and $\mathrm{pFC}$ complement each other in elucidating the bonding situation in materials like GeTe. We note that the two descriptors are entirely independent from each other and calculated from vastly different methodologies. While COBI (measuring the bond order) is extracted from the wavefunction, pFCs (measuring the bond stiffness) are derived perturbatively from atomic displacements. While bond strength and force constant often go parallel with each other, ${ }^{[33]}$ there is no obvious theoretical reason for them to be connected. And yet, both of them appear to resolve the same physical phenomenon for PCM, albeit in a different manner. One might argue that the relationship of the phenomena detected by COBI and pFC is one of cause (linear multicenter orbital interaction) and effect (unusually high force constants between these far-off atoms).

\subsection{Multicenter Bonding in Layered Materials}

If orbital-based COBI and lattice-dynamics based $\mathrm{pFC}$ arguments are valid for multicenter bonding in rocksalt type GeTe, they should also hold for other materials exhibiting the rocksalt motif given a similar valence-electron concentration. As alluded to before, such criteria also characterize prominent layered PCM like $\mathrm{Sb}_{2} \mathrm{Te}_{3}$ and the entire GST system, too. What will be the consequences of the limited chain length imposed by the terminal atoms (at the supposedly van-der-Waals gap) in these layered materials?

Figure 4 depicts the COBI plots and corresponding ICOBI values for different $\mathrm{Te} \stackrel{\mathrm{M}}{-} \mathrm{Te}$ threecenter interactions in various layered PCM as well as in rocksalt GeTe for comparison. Notably, as the GeTe content of the material increases and $\beta-G e T e$ is approached, the ICOBI value decreases gradually from an initial -0.158 in $\mathrm{Sb}_{2} \mathrm{Te}_{3}$ to the previously discussed -0.099 for 
GeTe. Considering the chemical nature of GST as a pseudobinary system of $\mathrm{Sb}_{2} \mathrm{Te}_{3}$ and $\mathrm{GeTe}$, this monotone shift is in line with chemical intuition because, as the $\mathrm{Ge} / \mathrm{Sb}$ ratio increases, the valence-electron concentration decreases and, as a consequence, so does the ICOBI (bond order). While the additional GeTe units in the system lower the electron count, they do not appear to significantly change the underlying multicenter nature of chemical bonding; not too surprisingly, the projected force constants for these compounds remain large regardless of the GeTe content. Alternatively expressed, atoms "sticking together" over large distances looks like a universal phenomenon in PCM and originating from multicenter bonding, and we reiterate that such behavior is well-known experimentally from atom-probe tomography. Nonetheless, there is also a gradual change in overall shape of the electronic structures (visualized by the COBI plots in Figure 4) because the distinct $\mathrm{Sb}_{2} \mathrm{Te}_{3}$-like appearance steadily transforms to resemble the one of GeTe as more GeTe is introduced. That is to say that the GeTe content of the GST system appears to control the electronic properties of the compound, ${ }^{[34]}$ but this effect does not fully translate into the chemical bonding mechanism. And yet, the most remarkable discovery in probing the chemical-bonding nature of the layered $\mathrm{Bi}_{2} \mathrm{Te}_{3}$-type PCM is the occurrence of multicenter interactions - apparent from both COBI and pFC - bridging the supposedly van-der-Waals-like gap between individual layers.

As an illustrative example, we take the binary $\mathrm{Sb}_{2} \mathrm{Te}_{3}$ and its quintuple layers whose three- and four-center interactions are depicted in Figure 5; exactly the same phenomenon is found for the other members of the GST system as well. Besides obvious three-center interactions within the layer (as also highlighted before for bulk GeTe), there is also a significant degree of bonding interaction in the three-center $\mathrm{Sb} \stackrel{\mathrm{TevdW}}{\mathrm{Te}}$ (in red) crossing the gap, an electronic communication between the layers which is apparent both from a non-zero COBI plot as well as significant ICOBI of -0.041 , about $1 / 4$ of the in-layer ICOBI. Similarly, the four-center

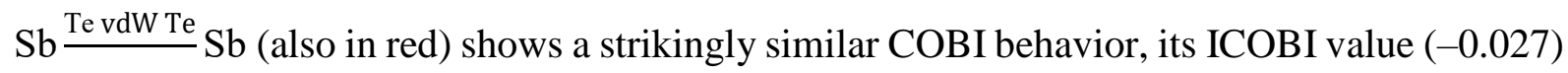


being almost as large as the three-center one, and it exhibits a force constant with a strength comparable to intralayer $\mathrm{Te} \stackrel{\mathrm{SbTe}}{\mathrm{Sb}}$, even stronger than the $\mathrm{pFC}$ for the three-center interaction across the gap. Evidently, multicenter interactions help maintaining the rocksalt structural motifs even across individual layers, and they provide an intuitive explanation as to the size of the gap between the layers. Multicenter orbital interactions make the gap shrink, significantly shorter than for regular van-der-Waals forces solely going back to vacuum fluctuations (and induced dipole-dipole interactions). Note that this has nothing to do with whatever a posteriori $\mathrm{vdW}$ correction to the exchange-correlation functional.

Mechanically, such gap-bridging interactions appear to differ slightly from those exclusively contained within a single layer, clearly visible in the smaller force constants and smaller ICOBI values for bridging interactions that end in a layer-terminating Te atom. This is likely connected to the break in the multicenter atomic sequence happening at the layer, with two subsequent $\mathrm{Te}$ atoms instead of the usual alternation, resulting in an electronically different four-center interaction. There is significant electronic localization at the gap with evidence for the presence of lone-pairs, ${ }^{[32]}$ making the presence of the strong force constants and ICOBI even more unusual.

\subsection{A Comprehensive Overview of Multicenter Interactions for all Compounds}

As presented before, upon going from the rocksalt type to layered compounds such as $\mathrm{Sb}_{2} \mathrm{Te}_{3}$, dismantling an extended structure's isotropy results in gradual changes as regards the multicenter bonding behavior which looks comparatively simple for the fundamental rocksalt systems. A detailed overview of the entire picture is given in Figure 6 in which projected force constants of multicenter interactions in a variety of compounds have been plotted against their corresponding integrated COBI values. Do these go together? 
A glancing look at Figure 6a makes it apparent that there is an obvious correlation between projected force constants (bond stiffness) and the crystal orbital bond index (bond order). Generally speaking, large force constant values correspond to a large (negative) multicenter ICOBI value, and vice versa. The figure also indicates, once again, that the bonding mechanism in the GST system does not qualitatively but only quantitatively change as the GeTe content increases. Irrespective of the actual composition, all equivalent multicenter indices and force constants are clustered together to such an extent that the attempt to make compositional differentiation seems difficult. Only the extreme system borders, that is, the binaries GeTe and $\mathrm{Sb}_{2} \mathrm{Te}_{3}$, are separated from the rest. This separation, however, is only observed in the projected force constant while the ICOBI values remain largely unchanged. As such, the difference in stiffness cannot originate from a difference in bonding mechanism.

Further, Figure $6 \mathrm{~b}$ demonstrates that the ICOBI values of the different equivalent three-center interactions are a consequence of the elemental nature of the bridging atom involved in $\mathrm{Te}-\mathrm{Ge}-\mathrm{Te}$, $\mathrm{Te} \stackrel{\mathrm{Sb}}{-} \mathrm{Te}$ and $\mathrm{M}-\mathrm{Te} \mathrm{M}$, and all three of them form separate clusters with nearly identical ICOBI values; clearly, the bridging atom (and its local electron count) is decisive for the three-center bond order. For $\mathrm{M}-\mathrm{Te}$, this cluster at reference point (RP) 1 is located closely around ICOBI $\approx 0$, largely independent of whether the precise motif is $\mathrm{Ge} \stackrel{\mathrm{Te}}{-} \mathrm{Sb}, \mathrm{Ge} \stackrel{\mathrm{Te}}{-} \mathrm{Ge}$ or $\mathrm{Sb} \stackrel{\mathrm{Te}}{-} \mathrm{Ge}$, a single exception being $\mathrm{Sb} \stackrel{\mathrm{Te}}{-} \mathrm{Sb}$ (appearing only in $\mathrm{Sb}_{2} \mathrm{Te}_{3}$ ) located comparatively far away at ICOBI $=-0.023$. We can safely conclude that these interactions involving a closed-shell atom (formally $\mathrm{Te}^{2-}$ with a noble-gas configuration) indicate an overall nonbonding state, as found before; even the outlier $\mathrm{Sb}-\mathrm{Te}$ Sb with the highest 5.6 valence-electron concentration (lowest triangle in the bluish cloud) only slightly shifts into the bonding regime. Quite to the contrary, $\mathrm{Te}-\underline{\mathrm{Ge}} \mathrm{Te}$ at RP 2 and $\mathrm{Te} \stackrel{\mathrm{Sb}}{-} \mathrm{Te}$ at RP 3 are outstanding and significant three-center interactions, mirroring the role of electron-rich bridging atoms (formally $\mathrm{Ge}^{2+}$ and $\mathrm{Sb}^{3+}$ with a surplus of two 
electrons outside the noble-gas shell), and this is where most of the multicenter bonding originates, as also detailed before.

Intralayer four-center interactions also form a cluster with lowered ICOBI and pFC (RP 4), just as expected. Once again, $\mathrm{Sb}_{2} \mathrm{Te}_{3}$ (RP 5) appears as an outlier, exhibiting a far weaker $\mathrm{pFC}$ for its four-center interaction. The gap-bridging four- and five-center interactions (RP 6) which lead to too short van-der-Waals contacts have comparably strong force constants but approach $\mathrm{ICOBI} \approx 0$ already for five centers. In contrast, all multicenter interactions that include a chainterminating Te atom have very small force constants and ICOBI.

Open questions remain, for example the occurrence of fairly weak five-center interactions with a positive ICOBI value (RP 7), yet to be explained. Even a simple chemical interpretation of such five-center index is challenging, as - to the best of our knowledge - no previous investigation of such a state has ever been tried. For a three-center case, positive values would indicate an electron-deficient multicenter bond but in the distinctly electron-rich systems investigated here, this is not a sensible interpretation. This is furthermore supported by their pFC which continue to fall and are the lowest for the largest positive ICOBI, which in turn is actually the most electron-rich compound $\mathrm{Sb}_{2} \mathrm{Te}_{3}$. We also note that some five-center interactions retain the their negative ICOBI sign, with GeTe and its Ge $\frac{\text { TeGeTe }}{\text { Ge interaction }}$ (RP 8) actually achieving force constants comparable to four-center interactions in the GST system. The range of the multicenter interaction could reasonably be interpreted as an indicator for the degree of electronic delocalization across the centers.

\section{Conclusion}

Given a tailored set of theoretical tools such as crystal orbital bond index and fragment crystal orbital analysis, an orbital-based study of the chemical bonding in the entire GST pseudobinary system has been carried out. In full accord with long-range projected force constants, there is 
clear evidence for electron-rich multicenter bonding for this class of compounds. To the best of our knowledge, such multicenter interactions have never before been investigated for periodic solids, the simple reason being that the aforementioned tools were unavailable so far.

Both electronic-structure (COBI) and phononic-structure ( $\mathrm{pFC}$ ) descriptors support multicenter bonding primarily via electron-rich intermediate atoms such as $\mathrm{Ge}$ or $\mathrm{Sb}$, to a smaller degree involving additional atoms, and even beyond van-der-Waals-like structural gaps. As such, the material properties portfolio usually related to metavalent bonding appear as a natural consequence of multicenter bonding, similar to the molecular case, but different due to the extended connectivity in the periodic solids. Not only does high delocalization across many centers naturally lead to an increased conductivity, the long-ranged forces between atoms “sticking together" as a consequence of multicenter bonding also serve as a simple explanation for the high probability of multiple emission events seen in atom-probe tomography. As alluded to before, structural gaps between layers are smaller than expected for van-der-Waals gaps, caused by the constructive atomic-orbital multicenter overlap between layers.

Unsurprisingly, considering the thorough phenomenological description of metavalent materials, a multicenter bonding mechanism as presented here also characterizes this very bonding type as being akin to both metallic and conventional (two-center) covalent bonding, exhibiting both a delocalized but also a directed character. While covalent bonding results from the interference of wavefunctions, such definition would strictly also include metallic bonding but one difference remains: although metallic bonding then appears as a special case of covalency, it is due to a too small number of electrons distributed over a plethora of atoms, hence completely delocalized across the bulk and therefore causing the characteristic metal properties. ${ }^{[35]}$ Curiously, the likewise interfering wavefunctions with too many electrons as discussed in this work yield the aforementioned peculiar PCM properties. That is to say that electron-rich multicenter bonding - just like electron-poor metallic bonding - may be understood as a special case of covalency. Supported by the amount of theoretical and also 
experimental data, we therefore conclude that multicenter bonding is in good agreement with the property-based concept of metavalent bonding, and we suggest multicenter bonding as the actual quantum-chemical origin of metavalency.

\section{Computational Details}

All structures were optimized using the Vienna ab initio Simulation Package (VASP). ${ }^{[36]}$ The PAW-based ${ }^{[37]}$ electronic wavefunctions were then projected onto a local orbital basis using the Local Orbital Basis Suite Towards Electronic-Structure Reconstruction (LOBSTER) package. ${ }^{[28]}$ As such, local quantities such as Mulliken/Löwdin populations ${ }^{[38]}$ and charges as well as bond orders as expressed by the crystal orbital bond index ${ }^{[13]}$ can be extracted from a plane-wave basis. All DFT simulations were considered as converged using criteria of $10^{-8} \mathrm{eV}$ for electronic and $5 \cdot 10^{-3} \mathrm{eV} / \AA$ for ionic iteration steps. The kinetic energy cutoff was set to $500 \mathrm{eV}$. PAW pseudopotentials were employed as recommended in the VASP manual. The Brillouin zone was sampled using k-point meshes of 0.02 to $0.04 \AA^{-1}$ and integrated with Blöchl's tetrahedron method ${ }^{[39]}$ using a smearing factor of $0.05 \mathrm{eV}$. The exchange-correlation interactions were treated within the generalized gradient approximation (GGA) as parametrized by Perdew, Burke and Ernzerhof ${ }^{[40]}$ with an additional van-der-Waals correction using the D3method with Becke-Johnson damping. ${ }^{[41]}$ The projected force constants were calculated from phononic $\Gamma$ point calculations on appropriately sized supercells using PHONOPY ${ }^{[42]}$ after the initial cell had been structurally optimized. The projection onto atomic pairs was executed as published previously. ${ }^{[12]}$ The fragment crystal orbital diagrams were constructed using the plane-wave eigenvalues from VASP as crystal orbital energies, the onsite Hamiltonian as atom/fragment orbital energies, and the LCAO coefficients for connectors between the energy levels, both of them extracted from LOBSTER projections. All values were averaged over the Brillouin zone. 

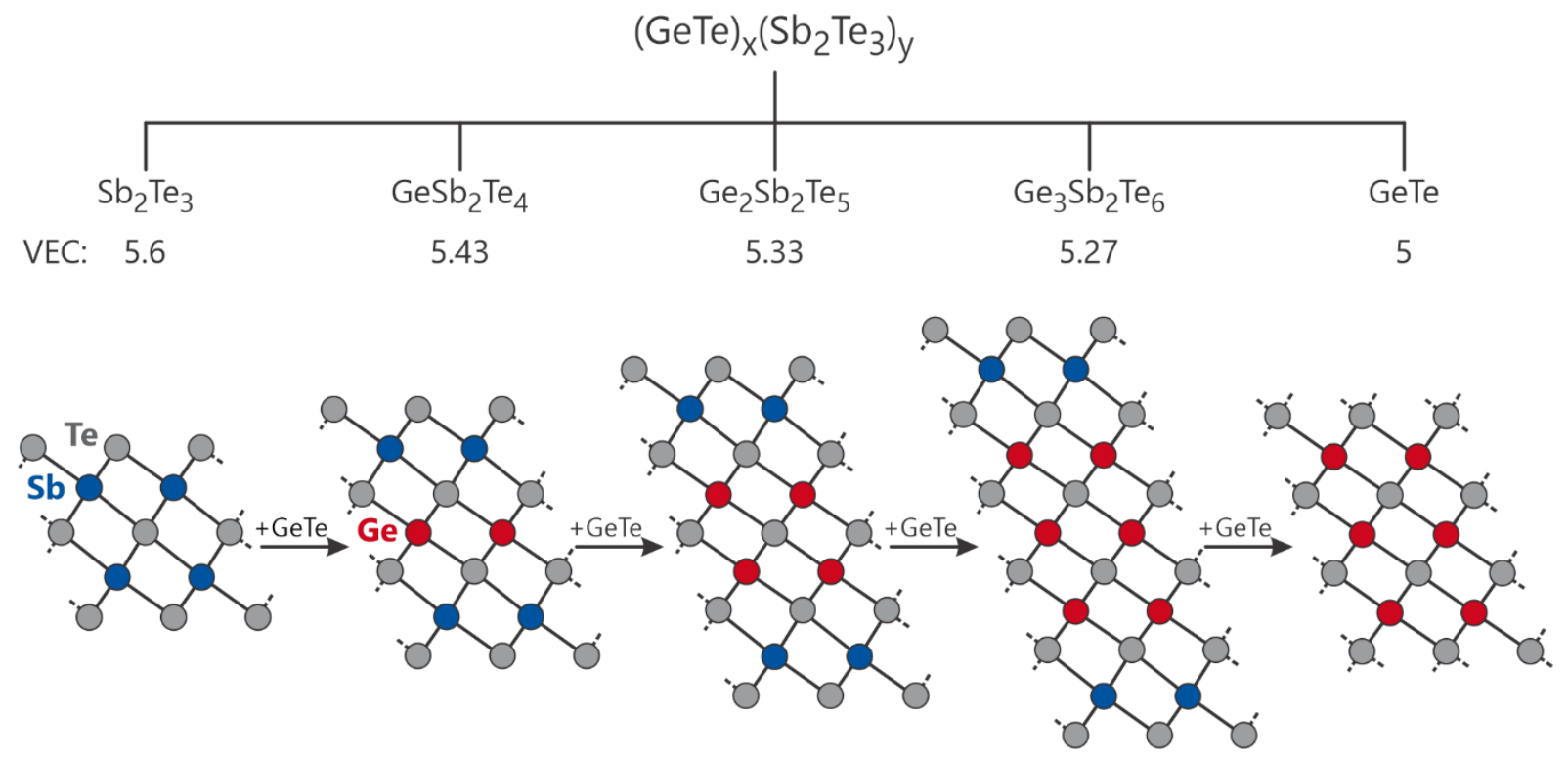

Figure 1 Schematic structural evolution of the GeTe- $\mathrm{Sb}_{2} \mathrm{Te}_{3}$ (“GST”) pseudo-binary system; starting from $\mathrm{Sb}_{2} \mathrm{Te}_{3}$ on the left, GeTe units are inserted between layer-terminating $\mathrm{SbTe}$ units. The end member on the right is rocksalt GeTe. The valence-electron concentration (= total valence-electron count divided by the number of atoms) lowers from left to right: 5.6, 5.43, $5.33,5.27$, and 5 .
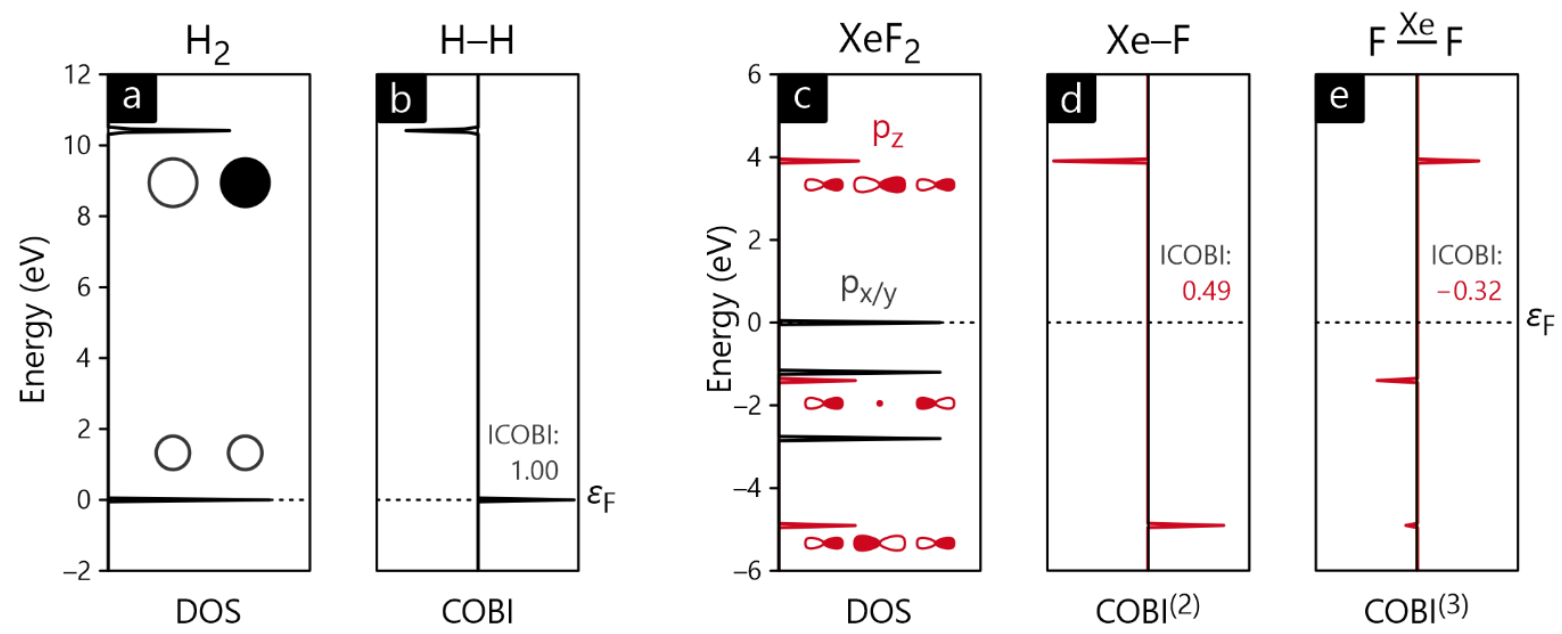

Figure 2 Molecular density of states (DOS) and crystal orbital bond index (COBI) plots for $\mathrm{H}_{2}$ (a, b), as well as DOS, two-center as well as three-center COBI plots for $\mathrm{XeF}_{2}$ (c, d, e). The molecular DFT calculations were carried out using periodic boundary conditions and plane waves (VASP), eventually projected to local atomic orbitals for final analysis (LOBSTER). 

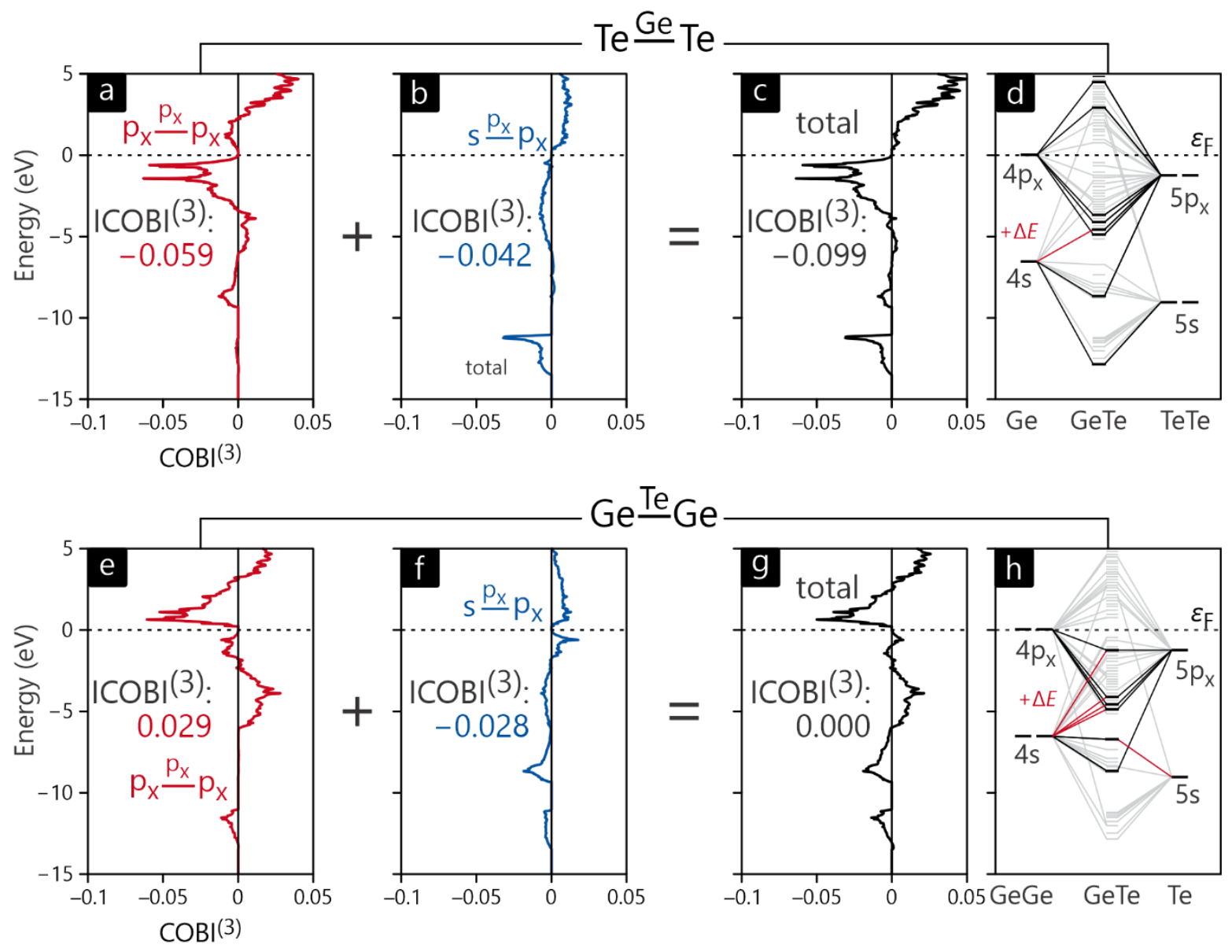

Figure 3 Orbitalwise and total three-center $\mathrm{COBI}$ plots for $\mathrm{Te} \stackrel{\mathrm{Ge}}{-\mathrm{Te}}(\mathbf{a}, \mathbf{b}, \mathbf{c})$ and $\mathrm{Ge} \stackrel{\mathrm{Te}}{-} \mathrm{Ge}(\mathbf{e}, \mathbf{f}$, g) as well as their respective fragment crystal orbital (FCO) diagrams (d) and (h). The corresponding integrated ICOBI values are given. Note that for Te $\stackrel{G e}{-} \mathrm{Te}$, the $\mathrm{p}_{\mathrm{x}} \stackrel{\mathrm{p}_{\mathrm{x}}}{\mathrm{p}_{\mathrm{x}}}$ and $\mathrm{s} \stackrel{\mathrm{p}_{\mathrm{x}}}{\mathrm{p}_{\mathrm{x}}}$ contributions add up to a total negative three-center ICOBI of approximately -0.10 while in $\mathrm{Ge} \stackrel{\mathrm{Te}}{-} \mathrm{Ge}$ they annihilate each other $(\mathrm{ICOBI}=0.00)$, indicative of a nonbonding state existing across several centers. 

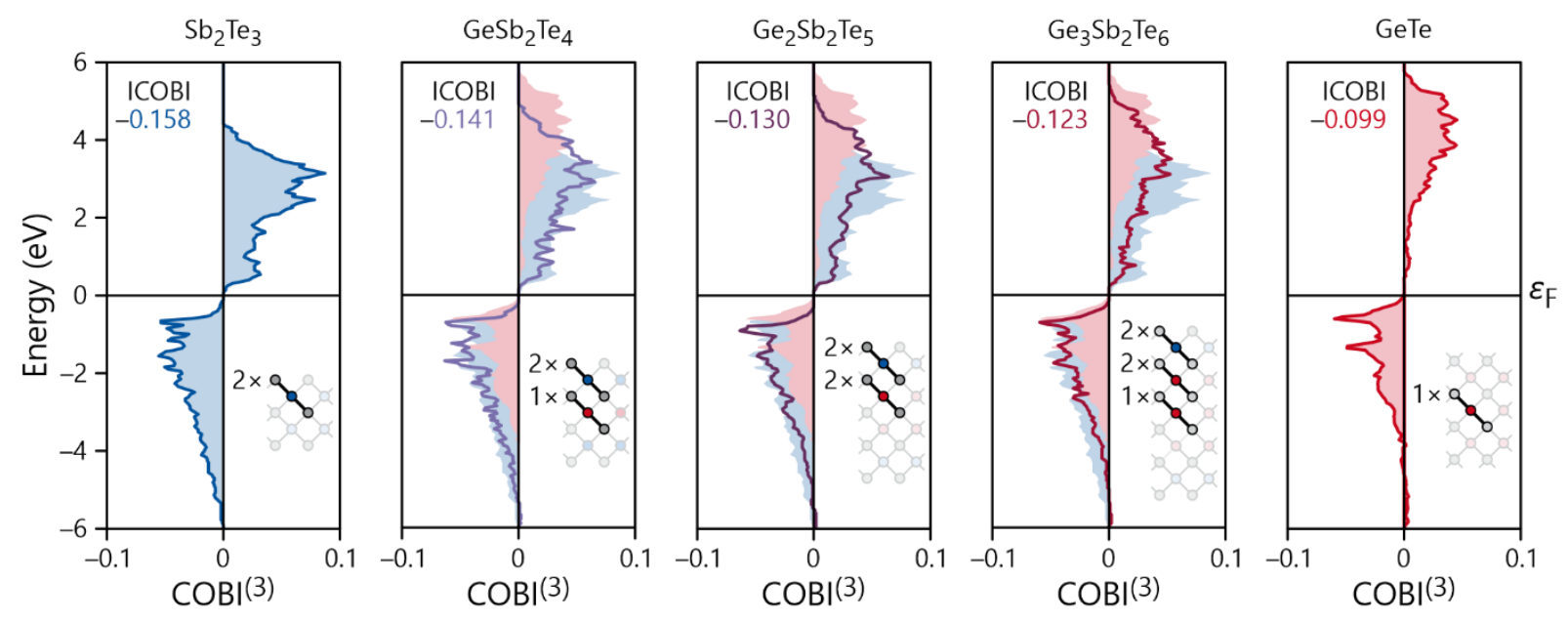

Figure 4 Averaged in-layer three-center COBI for $\mathrm{Sb}_{2} \mathrm{Te}_{3}$, GST124, GST225, GST326 and GeTe. Note how the COBI gradually shifts from an $\mathrm{Sb}_{2} \mathrm{Te}_{3}$ (blue) to a GeTe (red) nature. The pictograms refer to the different kinds of three-center interaction present in the respective system. 

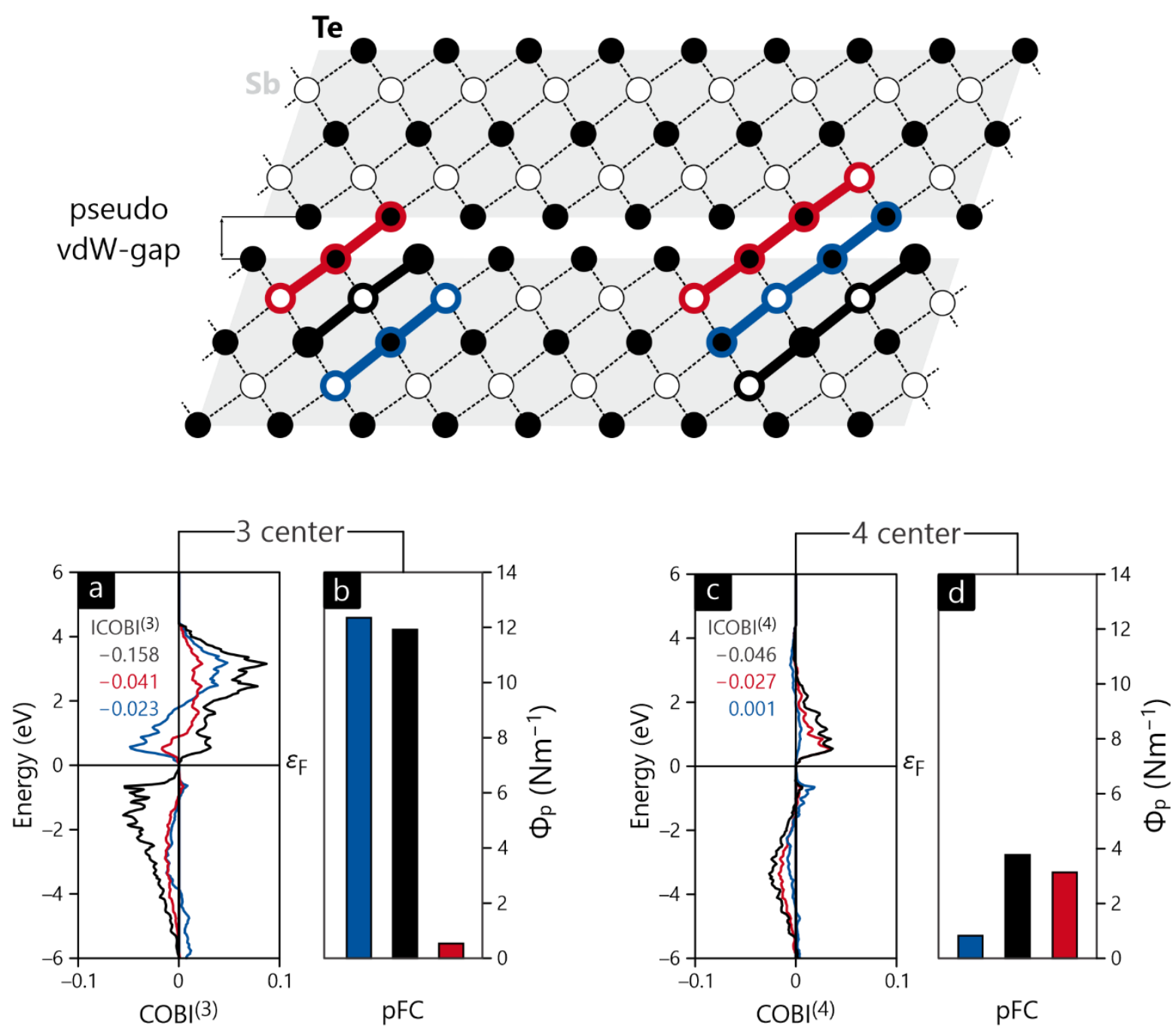

Figure 5 COBI plots $(\mathbf{a}, \mathbf{c})$ and projected force constants $(\mathbf{b}, \mathbf{d})$ for intra- and interlayer threeand four-center interactions in $\mathrm{Sb}_{2} \mathrm{Te}_{3}$. Note the significant values of both (I)COBI and $\mathrm{pFC}$

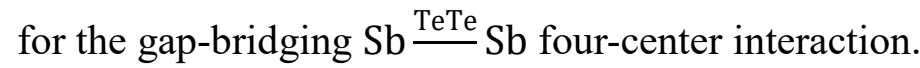




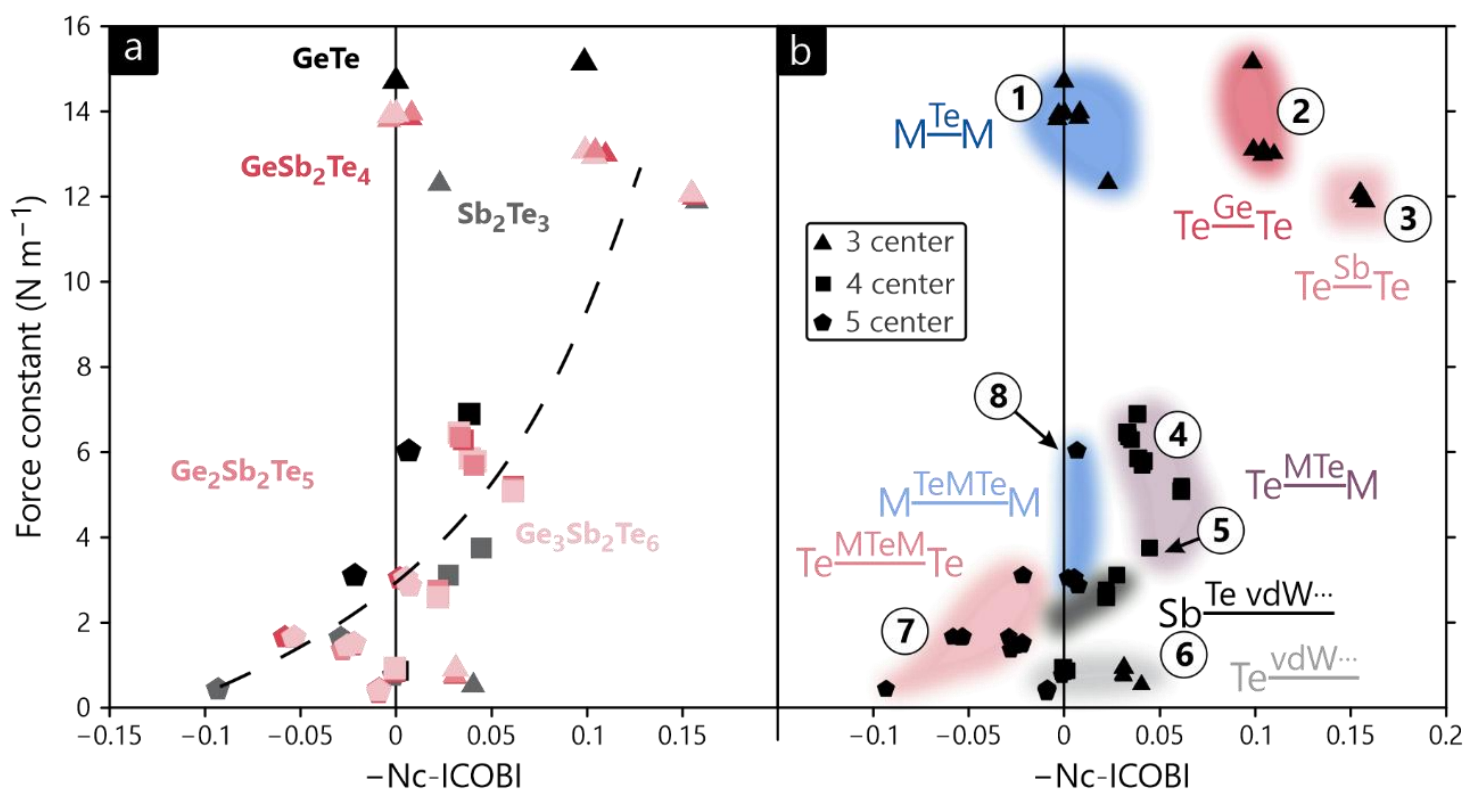

Figure 6 Projected force constants plotted against multicenter ICOBI for three-, four- and five-center interactions in GeTe, GST124, GST225, GST326 and $\mathrm{Sb}_{2} \mathrm{Te}_{3}$. In a) we show which data point belongs to which compound. In b), the data points are grouped according to the interaction type where $\mathrm{M}$ stands for either $\mathrm{Sb}$ or Ge, and vdW indicates an interlayer gapbridging interaction. For better orientation, reference points such as (1) have been sketched in b), please see text. 
J. Hempelmann, P. C. Müller, C. Ertural, R. Dronskowski* ((same order as byline))

\section{The Orbital Origins of Chemical Bonding in Phase-Change Materials}

ToC figure:

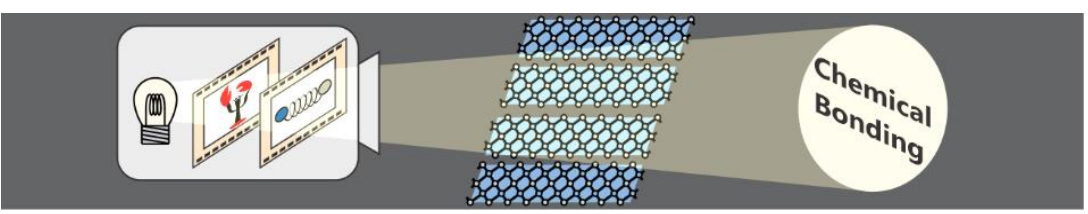

ToC text: Wavefunction analysis of phase-change materials in terms of interacting atomic orbitals reveals the decisive role of electron-rich multicenter interactions, similar yet different from the molecular case. The rather uncommon physical properties of these phases such as bond-breaking behavior and other structural peculiarities arise as a natural consequence of electron-rich multicenter bonding in condensed matter. 


\section{Acknowledgements}

P.C. Müller and J. Hempelmann contributed equally to this work. All authors gratefully acknowledge financial support obtained from Deutsche Forschungsgemeinschaft within SFB 917 "Nanoswitches". Also, we thank the Jülich-Aachen Research Alliance (JARA) as well as RWTH Aachen University's IT Center for having provided CPU time under project jara0033.

[1] M. G. Kanatzidis, Inorg. Chem. 2017, 56, 3158; C. Koch, G. Schienke, M. Paulsen, D. Meyer, M. Wimmer, H. Volker, M. Wuttig, W. Bensch, Chem. Mater. 2017, 29, 9320; M.

Wuttig, N. Yamada, Nat. Mater. 2007, 6, 824; S. Raoux, Annu. Rev. Mater. Res. 2009, 39, 25.

[2] S. Raoux, F. Xiong, M. Wuttig, E. Pop, MRS Bulletin 2014, 39, 703; S. R. Ovshinsky, Phys. Rev. Lett. 1968, 21, 1450; S. Raoux, W. Welnic, D. Ielmini, Chem. Rev. 2010, 110, 240; V. L. Deringer, R. Dronskowski, M. Wuttig, Adv. Funct. Mater. 2015, 25, 6343.

[3] L. Pauling, The Nature of the Chemical Bond, Cornell University Press, Ithaca, NY 1960.

[4] E. Mooser, W. B. Pearson, Acta Cryst. 1959, 12, 1015.

[5] B. J. Kooi, M. Wuttig, Adv. Mater. 2020, 32, 1908302.

[6] Y. Cheng, S. Wahl, M. Wuttig, Phys. Status Solidi RRL 2020, 15, 2000482; M.

Wuttig, V. L. Deringer, X. Gonze, C. Bichara, J. Y. Raty, Adv. Mater. 2018, 30, 1803777.

[7] M. Zhu, O. Cojocaru-Miredin, A. M. Mio, J. Keutgen, M. Küpers, Y. Yu, J. Y. Cho, R. Dronskowski, M. Wuttig, Adv. Mater. 2018, 30, 1706735.

[8] M. Küpers, R. P. Stoffel, B. Bong, M. G. Herrmann, Z. Li, A. Meledin, J. Mayer, K. Friese, R. Dronskowski, Z. Naturforsch. B 2020, 75, 41.

[9] J. Y. Raty, M. Schumacher, P. Golub, V. L. Deringer, C. Gatti, M. Wuttig, $A d v$. Mater. 2019, 31, 1806280.

[10] J. Hempelmann, P. C. Müller, P. M. Konze, R. P. Stoffel, S. Steinberg, R. Dronskowski, Adv. Mater. 2021, 33, 2100163.

[11] P. M. Konze, Dissertation, RWTH Aachen, 2019. 
[12] V. L. Deringer, R. P. Stoffel, M. Wuttig, R. Dronskowski, Chem. Sci. 2015, 6, 5255.

[13] P. C. Müller, C. Ertural, J. Hempelmann, R. Dronskowski, J. Phys. Chem. C 2021, $125,7959$.

[14] W. V. Glassey, G. A. Papoian, R. Hoffmann, J. Chem. Phys. 1999, 111, 893; W. V. Glassey, R. Hoffmann, J. Phys. Chem. B 2001, 105, 3245.

[15] T. Ohta, J. Optoelectron. Adv. Mater. 2001, 3, 609.

[16] S. A. Semiletov, Kristallografiya 1956, 1, 403.

[17] T. Matsunaga, R. Kojima, N. Yamada, K. Kifune, Y. Kubota, M. Takata, Appl. Phys. Lett. 2007, 90, 161919.

[18] K. Schubert, H. Fricke, Z. Naturforsch. A 1951, 6, 781.

[19] W. Heitler, F. London, Z. Phys. 1927, 44, 455.

[20] F. Hund, Z. Phys. 1926, 36, 657; R. S. Mulliken, Phys. Rev. 1928, 32, 186; J. P. Lowe, K. A. Peterson, Quantum Chemistry, Elsevier Academic Press, Burlington, MA 2006.

[21] W. N. Lipscomb, Science 1966, 153, 373.

[22] R. E. Rundle, J. Am. Chem. Soc. 1947, 69, 1327; R. E. Rundle, J. Chem. Phys. 1949, 17,671 .

[23] G. C. Pimentel, J. Chem. Phys. 1951, 19, 446.

[24] A. N. Alexandrova, A. I. Boldyrev, H.-J. Zhai, L.-S. Wang, J. Phys. Chem. A 2004, 108,3509 .

[25] M. L. Munzarová, R. Hoffmann, J. Am. Chem. Soc. 2002, 124, 4787.

[26] M. Giambiagi, M. S. de Giambiagi, K. C. Mundim, Struct. Chem. 1990, 1, 423; A. Sannigrahi, T. Kar, Chem. Phys. Lett. 1990, 173, 569.

[27] K. B. Wiberg, Tetrahedron 1968, 24, 1083; I. Mayer, Chem. Phys. Lett. 1983, 97, 270; I. Mayer, J. Comp. Chem. 2007, 28, 204.

[28] S. Maintz, V. L. Deringer, A. L. Tchougréeff, R. Dronskowski, J. Comput. Chem. 2013, 34, 2557; S. Maintz, V. L. Deringer, A. L. Tchougréeff, R. Dronskowski, J. Comput. Chem. 2016, 37, 1030; R. Nelson, C. Ertural, J. George, V. L. Deringer, G. Hautier, R. Dronskowski, J. Comput. Chem. 2020, 41, 1931. 
[29] P. C. Müller, Master Thesis, RWTH Aachen, 2019; P. M. Konze, Private communication.

[30] R. Hoffmann, H. Fujimoto, J. R. Swenson, C.-C. Wan, J. Am. Chem. Soc. 1973, 95, 7644.

[31] E. J. Skoug, D. T. Morelli, Phys. Rev. Lett. 2011, 107, 235901; M. D. Nielsen, V. Ozolins, J. P. Heremans, Energy Environ. Sci. 2013, 6, 570; S. Lee, K. Esfarjani, T. Luo, J. Zhou, Z. Tian, G. Chen, Nat. Commun. 2014, 5, 1; Y. Yu, M. Cagnoni, O. Cojocaru Mirédin, M. Wuttig, Adv. Funct. Mater. 2020, 30, 1904862; T. H. Lee, S. R. Elliott, Adv. Mater. 2020, 32, 2000340; T. H. Lee, S. R. Elliott, Phys. Status Solidi RRL 2021, 15, 2000516.

[32] J.-Y. Raty, C. Gatti, C.-F. Schön, M. Wuttig, Phys. Status Solidi RRL 2021, 2000534.

[33] L. Zhao, M. Zhi, G. Frenking, Int. J. Quantum Chem. 2021, 26773.

[34] A.-K. U. Michel, M. Wuttig, T. Taubner, Adv. Opt. Mater. 2017, 5, 1700261.

[35] W. P. Anderson, J. K. Burdett, P. T. Czech, J. Am. Chem. Soc. 1994, 116, 8808; L. C. Allen, J. K. Burdett, Angew. Chem. Int. Ed. 1995, 34, 2003.

[36] G. Kresse, J. Furthmüller, Comput. Mat. Sci. 1996, 6, 15; G. Kresse, J. Furthmüller, Phys. Rev. B 1996, 54, 11169; G. Kresse, J. Hafner, Phys. Rev. B 1993, 47, 558; G. Kresse, D. Joubert, Phys. Rev. B 1999, 59, 1758.

[37] P. E. Blöchl, Phys. Rev. B 1994, 50, 17953.

[38] C. Ertural, S. Steinberg, R. Dronskowski, RSC Adv. 2019, 9, 29821.

[39] P. E. Blöchl, O. Jepsen, O. K. Andersen, Phys. Rev. B 1994, 49, 16223.

[40] J. P. Perdew, K. Burke, M. Ernzerhof, Phys. Rev. Lett. 1996, 77, 3865; J. P. Perdew, W. Yue, Phys. Rev. B 1986, 33, 8800.

[41] S. Grimme, J. Antony, S. Ehrlich, H. Krieg, J. Chem. Phys. 2010, 132, 154104; S. Grimme, S. Ehrlich, L. Goerigk, J. Comput. Chem. 2011, 32, 1456.

[42] A. Togo, I. Tanaka, Scr. Mater. 2015, 108, 1. 